\title{
Late Multiple Stent Fractures Following Deployment of Sirolimus-Eluting Stents for Diffuse Right Coronary Artery Stenosis
}

\author{
Asako AoKI, ${ }^{1}$ MD, Jun Tanabe, ${ }^{1}$ MD, Toru Inami, ${ }^{1}$ MD, Michio Ogano, ${ }^{1}$ MD, \\ Nobuaki KoBAYASHI, ${ }^{1} \mathrm{MD}$, Yusuke HosOKAWA, ${ }^{1} \mathrm{MD}$, \\ Hiroyuki YokoYama, ${ }^{1} \mathrm{MD}$, Hitoshi TAKAnO, ${ }^{2} \mathrm{MD}$, and Kyoichi MizunO, ${ }^{2} \mathrm{MD}$
}

\begin{abstract}
SUMMARY
We report a case of late multiple stent fractures following the deployment of sirolimus-eluting stents (SESs) for diffuse right coronary artery (RCA) stenosis. A 44-year-old male with hyperlipidemia was referred to our hospital for acute myocardial infarction (AMI). Percutaneous coronary intervention (PCI) was performed for total occlusion of the proximal segment of the RCA, and 5 SESs were consecutively implanted for long, diffuse stenotic lesions. A follow-up coronary angiography (CAG) performed 8 months later revealed strut fractures in the middle of all the stents, except the most proximal one. Multislice computed tomography confirmed the locations of the fractured struts inside of the stents. (Int Heart J 2007; 48: 767-772)
\end{abstract}

Key words: Percutaneous coronary intervention, Drug-eluting stent, Stent strut fracture, Intravascular ultrasound, Multislice computed tomography

DRUG-eluting stents (DESs) have been used to address in-stent restenosis, a problem that has been plaguing coronary stenting for the past decade. DES implantations have dramatically reduced the incidence of in-stent restenosis, and a number of studies have shown better clinical outcomes following DES implantation when compared with bare-metal stent (BMS) implantation. ${ }^{1-2)}$ However, instent restenosis continues to occur after DES implantation in some cases, and its occurrence has been greatly responsible for limiting a patient's quality of life following percutaneous coronary intervention (PCI). Regarding sirolimus-eluting stents (SESs), previous trials have failed to show their advantage in preventing in-stent or peri-stent restenosis, particularly in patients with end-stage renal disease or insulin-dependent diabetes. ${ }^{3-4)}$ Thus, the problem of in-stent restenosis remains unresolved. In addition, an increasing number of stent fractures follow-

From the ${ }^{1}$ Division of Cardiology, Shizuoka Medical Center, Shimizu-cho, and ${ }^{2}$ Department of Medicine, Nippon Medical School, Tokyo, Japan.

Address for correspondence: Hitoshi Takano, MD, Department of Medicine, Nippon Medical School, 1-1-5 Sendagi, Bunkyo-ku, Tokyo 113-8603, Japan.

Received for publication July 19, 2007.

Revised and accepted November 6, 2007. 
ing DES implantations have been reported recently. ${ }^{5-13)}$ SESs, a DES with a rigid and inflexible platform, are closely associated with stent fractures. This new phenomenon is not a negligible issue since it may play a major role in the mechanism of restenosis after SES implantation. Here, we report a case of multiple stent fractures found 8 months after PCI with SES for diffuse right coronary artery (RCA) stenosis.

\section{CASE REPORT}

The patient was a 44-year-old male who was referred from a local general hospital to the coronary care unit of the Shizuoka Medical Center with a diagnosis of acute myocardial infarction (AMI). He had no past medical history or family history but he did have untreated hypertension and hyperlipidemia and a 24year history of smoking. He presented at the local hospital with sudden chest oppression. An electrocardiogram (ECG) showed a QS pattern in the precordial leads and negative T waves in leads V3-6 and Q waves in leads III and aVF. Coronary angiography $(\mathrm{CAG})$ revealed total occlusion of the proximal segment of the RCA, a subtotal occlusion in the mid portion of the left circumflex artery (LCX), and severe stenosis of the proximal segment of the left anterior descending artery (LAD) (Figure 1A). A transthoracic echocardiogram demonstrated hypokinesis of anteroseptal, apical and inferior wall segments and the LV ejection fraction was calculated to be $45 \%$. Since the local hospital was not equipped with a surgical team that could immediately attend to the patient in case of emergency, he was transferred to our hospital so as to undergo PCI in a steady condition, including surgical back up. Coronary artery bypass graft (CABG) surgery was recommended, but the patient declined the open-chest surgical treatment and opted for transluminal treatment. Elective PCI was performed 3 days postadmission.

First session of PCI: We presumed that the LAD lesion was the culprit lesion for the myocardial infarction since an ECG showed T wave inversion in the precordial leads. However, since the LAD was supplying the collateral flow to the distal segment of the RCA, the RCA lesion was treated first. The RCA was engaged with a 6 Fr guiding catheter (Launcher JR4), using a standard femoral approach. A balance universal guidewire was used to cross the lesion. After dilatation with a conventional angioplasty balloon, slow flow phenomenon occurred. Intravascular ultrasound (IVUS) showed massive soft plaque; therefore, stent implantation for the RCA was postponed, and the lipid-lowering agent atorvastatin $(40 \mathrm{mg} /$ day) was prescribed since his serum level of total cholesterol was $244 \mathrm{mg} / \mathrm{dL}$, triglycerides $191 \mathrm{mg} / \mathrm{dL}$, and LDL cholesterol $167 \mathrm{mg} / \mathrm{dL}$. The LAD lesions were subsequently treated. The guiding catheter was an 8 Fr mach1 CLS3.5, and the 


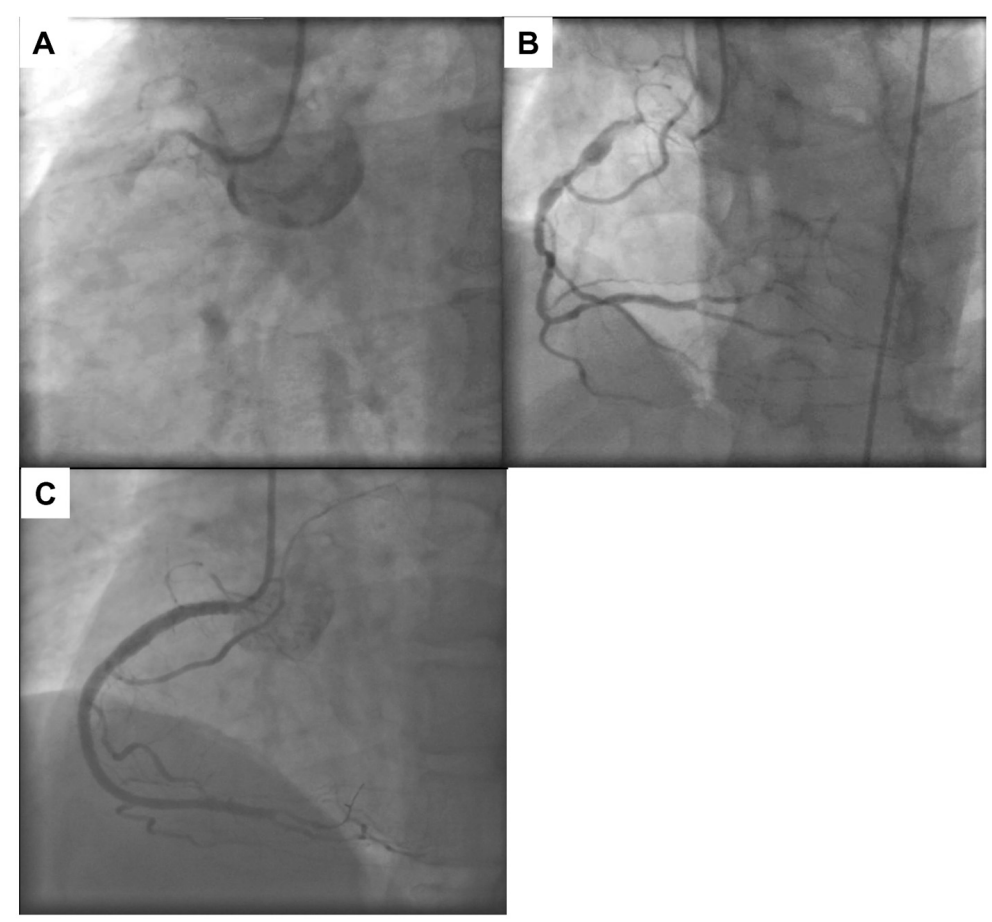

Figure 1. A: Right coronary angiography prior to percutaneous coronary intervention (PCI) revealed total occlusion of the proximal segment of the RCA. B: Right coronary angiography at 1 month after the first session. It was confirmed that long, diffuse complex lesions persisted. C: Right coronary angiography after the entire PCI procedure. Five sirolimus-eluting stents ( 2 of the $3.5-\mathrm{mm}$ and 3 of the $3.0-\mathrm{mm}$ types) were deployed, resulting in a good angiographic result.

approach was from the femoral artery. A Grand Slam guidewire was used to cross the lesion. IVUS demonstrated a lumen diameter greater than $4 \mathrm{~mm}$; therefore, the plaque was debulked at the proximal lesion by a directional coronary atherectomy (DCA). After dilatation with a conventional angioplasty balloon, a DCA was performed for the proximal lesion, resulting in a wide lumen area. A stent was not deployed for this lesion. For the stenotic lesion at the mid portion of the LAD, a 3.0-mm diameter Cypher was deployed after predilatation, and optimal dilatation was obtained.

Second session of PCI: One month later, CAG was repeated in order to attempt a PCI for the residual stenosis of the RCA. The CAG confirmed that long, diffuse complex lesions remained and also showed no restenosis in the sites treated with DCA or in the LAD stent (Figure 1B). The RCA osmium lesion was treated first. After predilatation with a 3.5-mm conventional angioplasty balloon, an SES (3.5 $\times 23 \mathrm{~mm}$ ) was deployed. The remaining stenotic lesions were treated with SES implantation after predilatation with a conventional angioplasty balloon $(3.5 \times 20$ 
$\mathrm{mm})$. Two Cypher stents $(3.0 \times 28 \mathrm{~mm})$ were deployed to the distal stenotic lesions, 1 Cypher stent $(3.0 \times 18 \mathrm{~mm})$ to the middle lesion, and 1 Cypher stent $(3.5 \times 23 \mathrm{~mm})$ to the proximal lesion. The proximal stent was dilated with a highpressure 4.0-mm balloon. During the entire session, a total of 5 SESs (2 of the $3.5-\mathrm{mm}$ and 3 of the $3.0-\mathrm{mm}$ types) were deployed to the RCA, resulting in a good and immediate angiographic result (Figure 1C).

Follow-up CAG: The patient remained asymptomatic after the PCI sessions. A follow-up CAG was performed 8 months after SES deployment. RCA angiography showed a stent strut deficit in the middle portion of 3 of the SESs; late fracture of the stents was indicated. The most distal stent appeared to be intact. Each of the 3 stents was observed to be fractured in its mid portion - the point of sharp vessel curvature and maximal movement. Intravascular ultrasound confirmed disruption of the strut at every part corresponding to the fracture site, and some degree of intimal hyperplasia was observed at the gap between the fractured struts (Figure 2). A subsequent multislice coronary CT (MSCT) performed after confirming the stent fracture demonstrated that 4 of the 5 stents were fractured in the

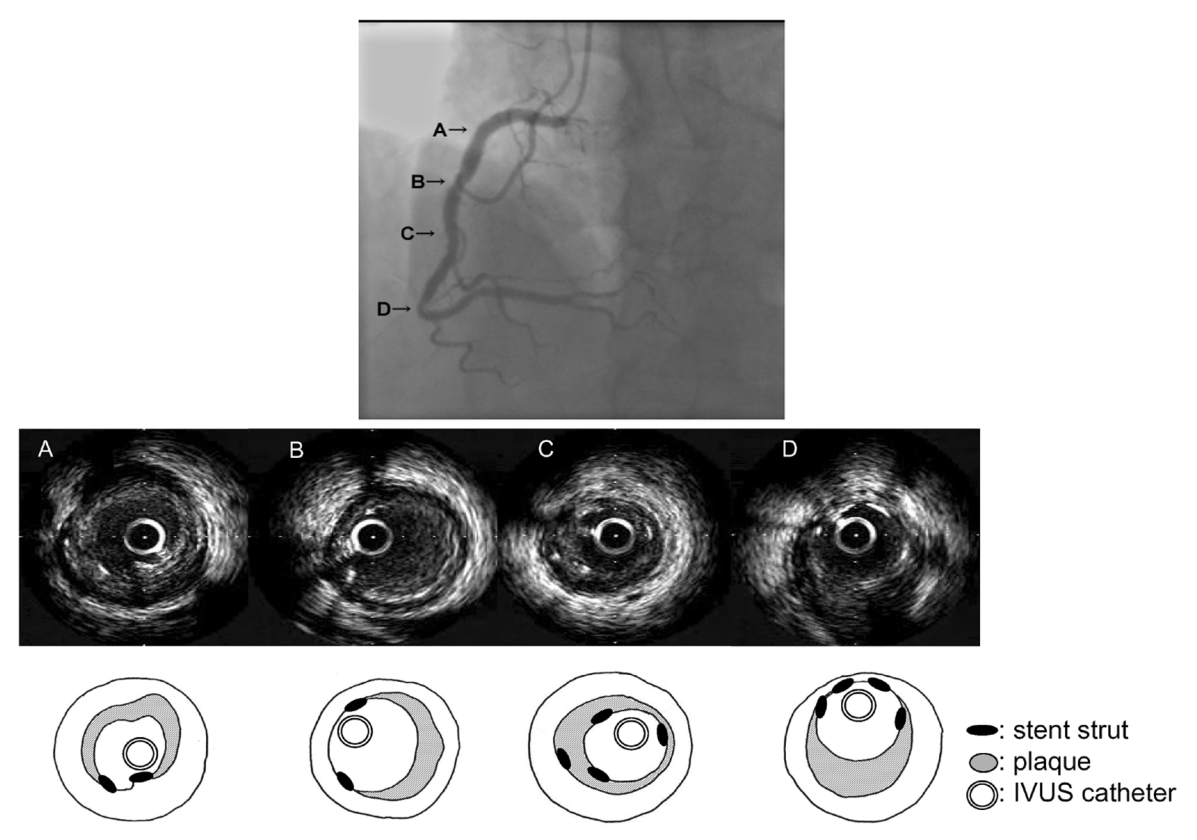

Figure 2. Images of intravascular ultrasonography of right coronary artery 8 months after PCI (A-D) in the middle panel. A: an image at the fracture site in the most proximal stent showing the disruption of strut. B: an image in the proximally second stent. C: an image in the proximally third stent. D: an image in the distally second stent. The images correspond to the stent fracture sites shown in the right coronary arteriography (Upper). Sketches corresponding to the IVUS images are shown in the lower panel. Each image reveals the disruption of the stent and the development of intimal hyperplasia where the stent strut was absent. 


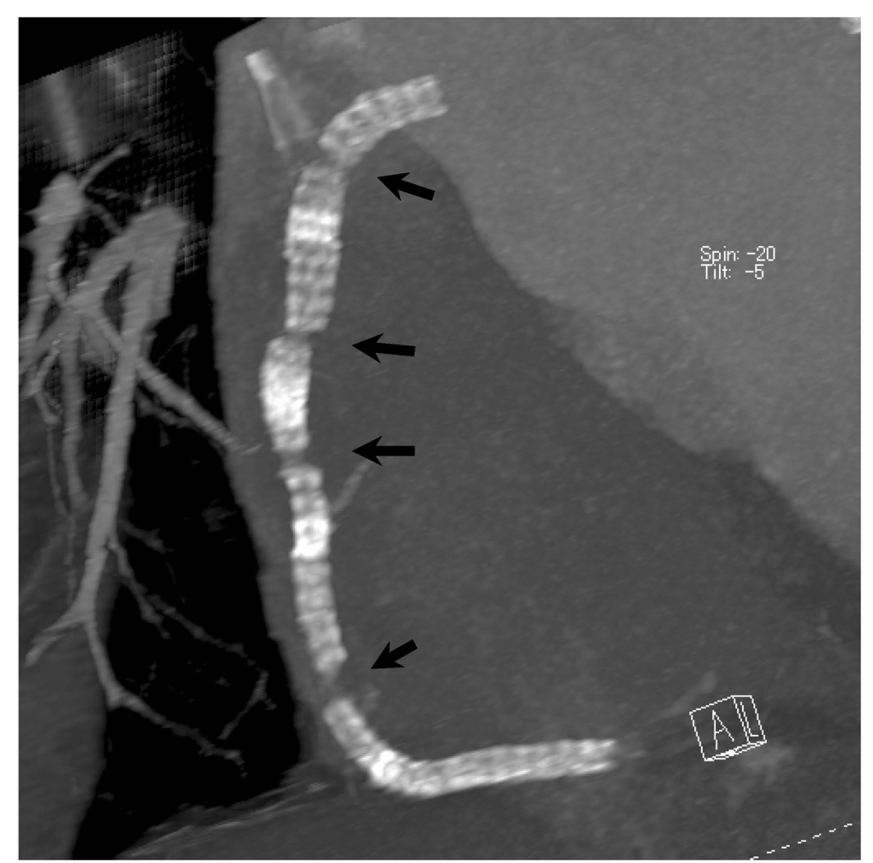

Figure 3. Curved multiplanar reconstruction image of RCA in multislice computed tomography at 8 months after PCI. A complete strut fracture was found at the middle of the 4 sirolimuseluting stents, with the exception of the distal one. Arrows indicate the sites of stent fracture.

middle and that each fractured part corresponded with the sharp point of the curvature (Figure 3).

\section{DISCUSSION}

An increasing number of stent fractures have been reported in the era of DESs. The incidence of stent fracture after SES implantation is reported to be $2.6 \% .{ }^{4)}$ Although the mechanism underlying these stent fractures has not been completely elucidated, several possible explanations have been suggested. Metal overlap and vessel movement during cardiac contraction may induce a mechanical force against the implanted stent, thereby causing fracture. ${ }^{5,7)}$ Stent expansion due to postdilatation with a larger, high-pressure balloon is believed to be a factor as well. ${ }^{7,8)}$ We speculate that metal fatigue at the hinge point in the sharp curvature of the stent plays the most important role in the development of this phenomenon. In the case presented here, the implantation of multiple and overlapping stents restricted the flexibility and increased the local rigidity of the stents, maximizing 
the distortion at the adjacent parts. The stent fracture occurred due to metal fatigue from excessive mechanical force at the hinge points during the vessel movement associated with cardiac contraction.

The management of stent fracture continues to remain controversial, and consensus regarding the best approach to manage stent fractures has not been reached. Similar to in-stent restenosis, the probability of re-restenosis is considered to be high if treated with balloon angioplasty or a BMS implantation. Implantation with another SES may result in refracture. The use of paclitaxeleluting stents or other newly developing DESs might be an appropriate selection since the stent platform is more flexible than the SES. ${ }^{11)}$ In the present case, the lesions were not treated percutaneously because the severity of restenosis was mild and myocardial ischemia was not apparent.

We observed multiple stent fractures following the implantation of multiple stents in the RCA. A follow-up MSCT clearly demonstrated that the fractures occurred at the hinge points in the middle of the stents. Stent strut fracture is one of the most pressing issues in the DES era since these fractures are unpredictable and difficult to treat once restenosis occurs at the fracture.

\section{REFERENCES}

1. Morice MC, Serruys PW, Sousa JE, et al. A randomized comparison of a sirolimus-eluting stent with a standard stent for coronary revascularization. N Engl J Med 2002; 346: 1773-80.

2. Moses JW, Leon MB, Popma JJ, et al. Sirolimus-eluting stents versus standard stents in patients with stenosis in a native coronary artery. $\mathrm{N}$ Engl $\mathrm{J}$ Med 2003; 349: 1315-23.

3. Nakazawa G, Tanabe K, Aoki J, et al. Clinical and angiographic outcomes of sirolimus-eluting stents implantation in Japanese patients in daily practice. Circ J 2006; 70: 1367-71.

4. Aoki J, Nakazawa G, Tanabe K, et al. Incidence and clinical impact of coronary stent fracture after sirolimuseluting stent implantation. Catheter Cardiovasc Interv 2007; 69: 380-6.

5. Kang WY, Kim W, Kim HG, Kim W. Drug-eluting stent fracture occurred within 2 days after stent implantation. Int J Cardiol 2007; 120: 273-5.

6. Lee MS, Jurewitz D, Aragon J, Forrester J, Makkar RR, Kar S. Stent fracture associated with drug-eluting stents: clinical characteristics and implications. Catheter Cardiovasc Interv 2007; 69: 387-94.

7. Sianos G, Hofma S, Ligthart JM, et al. Stent fracture and restenosis in the drug-eluting stent era. Catheter Cardiovasc Interv 2004; 61: 111-6.

8. Halkin A, Carlier S, Leon MB. Late incomplete lesion coverage following Cypher stent deployment for diffuse right coronary artery stenosis. Heart 2004; 90: e45.

9. Min PK, Yoon YW, Moon Kwon H. Delayed strut fracture of sirolimus-eluting stent: a significant problem or an occasional observation? Int J Cardiol 2006; 106: 404-6.

10. Lemos PA, Saia F, Ligthart JM, et al. Coronary restenosis after sirolimus-eluting stent implantation: morphological description and mechanistic analysis from a consecutive series of cases. Circulation 2003; 108: 257-60.

11. Kim EJ, Rha SW, Wani SP, et al. Coronary stent fracture and restenosis in the drug-eluting stent era: do we have clues of management? Int J Cardiol 2007; 120: 417-9.

12. Surmely JF, Kinoshita Y, Dash D, et al. Stent strut fracture-induced restenosis in a bifurcation lesion treated with the crush stenting technique. Circ J 2006; 70: 936-8.

13. Zaizen H, Tamura A, Miyamoto K, Nakaishi T, Kadota J. Complete fracture of sirolimus-eluting stent detected by multislice computed tomography. Int J Cardiol 2007; 118: 120-1. 